\title{
The use of tranexamic acid in elective lung surgery: a single- center experience
}

\author{
Ayman A. Sallam ${ }^{\mathrm{a}}$, Mohamed M. Abo El Nasr ${ }^{\mathrm{a}}$, Ahmed S. Elgebaly ${ }^{\mathrm{b}}$, \\ Wael M. El Fekyc
}

\begin{abstract}
Context Acute pulmonary embolism (PE) is a lethal sequela of venous thromboembolism (VTE). Surgical trauma injures the tissue directly, releasing a large number of tissue agent. The frequency of re-exploration owing to bleeding after lung surgery is between 1 and $3.7 \%$, whereas the need of allogenic blood transfusion spans from 20 to $52 \%$.
\end{abstract}

Aims To assess the role of tranexamic acid (TXA) in reducing the need of allogenic blood transfusion in patients undergoing elective lung surgery.

Patients and methods This retrospective study was conducted on 140 patients who underwent elective lung surgery. Patients were allocated into two groups. Group I patients received TXA at the end of procedure, and group II patients received blood and/or blood product transfusion.

Statistical analysis Qualitative variables are expressed as mean \pm SD. Quantitative variables are compared by using the Student's $t$ test.

Results The patients comprised 80 males and 60 females in our series. All cases in group II needed transfusion of one or more of the following: concentrated red blood cells, whole blood, fresh frozen plasma, and platelets. There was an

\section{Introduction}

Acute pulmonary embolism (PE) is a lethal sequela of venous thromboembolism (VTE), which if not actively suspected and adequately treated might be a sufficient cause of death. The incidence of deep vein thrombosis (DVT) is more than $20 \%$ in cases undergoing major surgery, even more up to $40 \%$ in serious orthopedic surgeries [1]. Postoperative PE may reach $5 \%$ in some patients subjected to a high thrombotic danger [2]. PErelated mortality reaches up to $30 \%$ in neglected patients with $\mathrm{PE}$, whereas it can be as low as $2-10 \%$ in timely managed patients; nevertheless, it is the most widely recognized reason of preventable death in hospital [3]. Surgical trauma injures the tissue directly, releasing an enormous amount of tissue agents, which enter the circulation in a brief time and initiate the exogenous coagulation system. Surgical operation also destructs and exposes elements such as subcutaneous collagen; therefore, endogenous coagulation cascade also becomes activated [4]. On the contrary, exposure of serous membranes like pleura, peritoneum, and pericardium to surgical insult liberates a significant amount of plasminogen activators that increase the local fibrinolytic activity and lead to more shedding of blood [5,6]. The frequency of re-exploration for bleeding after thoracic surgery is between 1 and obvious decrease in the postoperative hemoglobin level between groups, in favor of blood transfusion group (group II), and this was statistically significant.

Conclusions Elective thoracic surgery patients have a low incidence of VTE and PE (2.85 and 2.14\%, respectively). Hence, TXA helps in minimizing not only transfusion-related hazards but also operative cost.

Egypt J Bronchol 2019 13:699-703

(C) 2020 Egyptian Journal of Bronchology

Egyptian Journal of Bronchology 2019 13:699-703

Keywords: elective lung surgery, single-center experience, tranexamic acid

Departments of, a Cardio-thoracic Surgery, ${ }^{b}$ Anesthesia and Post-Surgical Intensive Care, Tanta University, Tanta, 'Department of Cardiothoracic Surgery, Kafr Elsheikh University, Kafr Elsheikh, Egypt

Correspondence to Ayman A. Sallam, MD, Department of Cardio-thoracic Surgery, Tanta University, Tanta, Egypt. Tel: +20 109812 1122; e-mail: ayman_sal@hotmail.com

Received: 6 April 2019 Accepted: 10 September 2019 Published: 21 January 2020

$3.7 \%$, whereas the need of allogenic blood transfusion spans from 20 to $52 \% \quad[7,8]$. As perioperative blood loss and anemia might be the leading causes of adverse outcomes after surgery, they were often treated with blood transfusion. Previous studies $[9,10]$ have shown tranexamic acid (TXA), as a synthetic antifibrinolytic agent, has significant hemostatic activity in all patients undergoing cardiovascular surgery. Based on its proven hemostatic properties, we were encouraged to use TXA in blood-sparing policy for elective lung surgery patients at our center. On the contrary, published data [11] found that blood transfusion itself was correlated with adverse outcomes such as infection, thromboembolism, respiratory failure, kidney failure, and cardiac-related hospital mortality. Therefore, the aims of our study are to (a) assess the role of TXA in decreasing the need of allogenic blood transfusion in patients undergoing elective lung operation and (b) identify the incidence and risk factors of PE including TXA and allogenic blood

This is an open access journal, and articles are distributed under the terms of the Creative Commons Attribution-NonCommercial-ShareAlike 4.0 License, which allows others to remix, tweak, and build upon the work non-commercially, as long as appropriate credit is given and the new creations are licensed under the identical terms. 
transfusion in patients undergoing elective lung surgery.

\section{Patients and methods}

Our study is a retrospective, single-center experience. Data were collected from patients underwent conventional lung resection surgery from July 2016 to July 2018. Its retrospective study and there is no consent. This retrospective study was conducted on 140 patients in the Cardio-thoracic Surgery Department, Tanta University Hospital. For suspected postoperative DVT cases, such as unexplained dyspnea, hemoptysis, chest pain, and highly suspected PE, bilateral lower limbs duplex venous scan after surgery for exclusion or confirmation of DVT, was done. Further, computed tomography pulmonary angiography was requested. The research has been approved by a research ethics committee.

Data collection included coagulation profile and all parameters related to fibrinolysis, such as antithrombin, fibrinogen degradation product, prothrombin time, prothrombin time activity, international normalized ratio, activated partial thromboplastin, and D-dimer. Patients were subjected to intense postoperative respiratory physiotherapy, which included early ambulation. After discharge, all patients were re-evaluated in the outpatient clinic after 1 month of surgery. Total blood loss estimation was done on the first postoperative day, and bleeding was defined as exaggerated if increased more than $600 \mathrm{ml} / 24 \mathrm{~h}$. Chest tubes were extracted when daily drainage was $100 \mathrm{ml}$ or less. Surgical re-exploration was considered when drainage exceeded $300 \mathrm{ml} / \mathrm{h}$ in the first two postoperative hours, or if more than $200 \mathrm{ml} / \mathrm{h}$ for four consecutive hours, provided that all coagulation parameters are normal. Blood transfusion conservative protocol was initiated by giving TXA in a dose of $50 \mathrm{mg} /$ $\mathrm{kg}$ at the end of surgery as a bolus dose and could be repeated $25 \mathrm{mg} / \mathrm{kg}$ if needed in an attempt to avoid blood and blood product transfusion (BT). The increased dose of TXA in our study was suggested by Mosaad et al. [12] who used a protocol in which prophylactic dose of TXA $(50 \mathrm{mg} / \mathrm{kg}$ loading dose then $20 \mathrm{mg} / \mathrm{kg}$ maintenance dose) provides a significant and focused strategy method for lowering perioperative and postoperative blood loss in thoracic spinal operations. Our patients were allocated into two groups based on whether they received TXA or not: group I patients received TXA at the end of procedure, and group II patients received blood and/or BT (perioperative and/or postoperative).

\section{Variables}

Analyzed variables included demographic data (age and sex), preoperative data like BMI, and intraoperative data like type of surgery (major/ minor). Time of surgery and time of anesthesia (beginning from skin cut till the removal of the surgical coverings and the time from inducing anesthesia to the minute that the patient is awaken, respectively) were noted as well. Hemoglobin concentration was estimated preoperatively and 1 day postoperatively. We defined intraoperative and/ or postoperative blood transfusion as the amount of blood or blood products transfused to the patient starting from the moment the patient goes into the operating room (intraoperative) and up to 3 days ( $72 \mathrm{~h}$ ) postoperatively. The amount of intraoperative blood loss was determined by including the measure of blood in the suckers to the weight of the used sponges. All fluids added to the surgical field were determined and deducted from the overall amount of blood loss.

\section{Inclusion criteria}

All available data and detailed medical records of consecutive patients underwent conventional lung resection without preoperative prophylaxis of VTE were included.

\section{Exclusion criteria}

We excluded patients with preoperative anticoagulation treatment like heparin, warfarin, and aspirin; patients who were diagnosed as having DVT or PE preoperatively; and patients with incomplete, inadequate, or missing data, especially those who did not go through VTE concomitant imaging study, coagulation profile, and fibrinolysis blood test preoperatively or postoperatively. This study, however, excluded patients with renal and hepatic failure (and potentially at a higher risk of bleeding).

\section{Outcome measurements}

Primary outcomes included first postoperative blood loss and unplanned reoperation for bleeding. Secondary outcomes included persistent air leak, empyema/bronchopleural fistula, chest tube indwelling time (days), length of hospital stay (days), acute renal failure, any thromboembolic events, and mortality (hospital/after discharge).

\section{Statistical analysis}

All variables were collected together in a computer database for analysis using the SPSS statistical package (SPSS Inc., Chicago, Illinois, USA). Qualitative variables are expressed as mean $\pm \mathrm{SD}$, unless abnormal 
distribution, in which case, they are expressed as median and range. Quantitative variables are compared by using the Student's $t$ test. The variable differences are considered statistically significant if the $P$ value is less than or equal to 0.05 .

\section{Results}

In the present study, a retrospective analysis was performed on the clinical data of 140 consecutive patients undergoing routine open thoracotomy for lung resection surgery and comprised the study population at Cardio-thoracic Surgery Department, Tanta University Hospital.

The patients comprised 80 males and 60 females in our series. There were 98 never smokers, 13 ex-smokers, and 29 current smokers. Ex-smokers were known as those who stopped smoking three years before the surgery time. All cases in group II needed transfusion of one or more of the following; concentrated red blood cells, whole blood, fresh frozen plasma, and platelets.
In Table 1, a summary of the characteristics of all patients is given, and there are no significant differences between both groups regarding age, sex, BMI, preoperative lung function test, and preoperative hemoglobin and smoking status.

A surgical team that included at least one surgical consultant performed all surgeries. Concerning the intraoperative factors, there were no significant differences in the approach, lesion site, procedure, or surgeon (Table 2). Nevertheless, no significant correlations was noticed between the incidence of postoperative bleeding and age, sex, BMI, preoperative lung function test, and preoperative hemoglobin and smoking status.

There was an obvious decrease in the postoperative hemoglobin level between groups, in favor of blood transfusion group (group II), and this was statistically significant (Table 3). Not all patients were successfully discharged from the hospital, as two patients died, one in each group, without any significant differences

Table 1 Clinical characteristics of patients in both groups (total number $=140$ patients: group I=65 and group II=75)

\begin{tabular}{lccc}
\hline Variables & Group I $(N=65)$ TXA group & Group II $(N=75)$ BT group & $P$ value \\
\hline Age at operation & $46.05 \pm 12.04$ & $44.35 \pm 12.2$ & 0.89 \\
Sex: female & $28(43.07)$ & $32(42.6)$ & 0.35 \\
BMI $\left(\mathrm{kg} / \mathrm{m}^{2}\right)$ & $29.9 \pm 3.5$ & $29.8 \pm 3.2$ & 0.99 \\
Smoking status & & & \\
$\quad$ Current & $15(23.07)$ & $14(18.6)$ & 0.09 \\
EX-smoker & $5(7.7)$ & $8(10.6)$ & 0.11 \\
Nonsmoker & $45(69.2)$ & $53(70.6)$ & 0.72 \\
Preoperative LFT & & & 0.66 \\
FEV1 & $2.37 \pm 0.57$ & $2.33 \pm 0.66$ & 0.91 \\
FCV & $3.34 \pm 0.76$ & $3.35 \pm 0.86$ & 0.26 \\
FEV1/FCV & $71.57 \pm 10.15$ & $-70.54 \pm 8.65$ & 0.4175 \\
Preoperative Hb & $13.1 \pm 1.16$ & $13.2 \pm 0.89$ & \\
\hline
\end{tabular}

BT, blood product transfusion; FEV1, forced expiratory volume; FVC, forced vital capacity; Hb, hemoglobin; LFT, lung function tests; TXA, tranexamic acid. Data were expressed as mean $\pm S D$ or frequency as percentage.

Table 2 Intraoperative characteristics of patients in both groups (total number=140 patients: group I=65 and group II=75)

\begin{tabular}{lccc}
\hline Variables & Group I $(N=65)$ TXA group & Group II $(N=75)$ BT group & $P$ value \\
\hline Operative time $(\mathrm{min})$ & $119.1 \pm 15.5$ & $125.4 \pm 12.5$ & 0.09 \\
Anesthesia time $(\mathrm{min})$ & $145.5 \pm 6.3$ & $155.4 \pm 4.1$ & 0.48 \\
Type of resection & & & \\
Wedge resection & $2(3.07)$ & $3(4.0)$ & 0.78 \\
RUL & $22(33.8)$ & $22(29.3)$ & 0.07 \\
RML & $4(6.1)$ & $5(6.6)$ & 0.83 \\
RLL & $13(20)$ & $16(21.3)$ & 0.97 \\
LUL & $12(18.4)$ & $13(17.3)$ & 0.89 \\
LLL & $9(13.8)$ & $12(16.0)$ & 0.08 \\
Pneumonectomy & $3(4.6)$ & $4(5.3)$ & 0.71 \\
\hline
\end{tabular}

BT, blood product transfusion; LLL, left lower lobe; LUL, left upper lobe; RLL, right lower lobe; RML, right middle lobe; RUL, right upper lobe; TXA, tranexamic acid. Data expressed as mean \pm SD or frequency as percentage. 
Table 3 Postoperative characteristics of patients in both groups (total number $=\mathbf{1 4 0}$ patients: group I=65 and group II=75)

\begin{tabular}{lccc}
\hline Variables & Group I $(N=65)$ TXA group & Group II $(N=75)$ BT group & $P$ value \\
\hline Blood loss during day $1(\mathrm{ml})$ & $382.50 \pm 119.5$ & $552 \pm 164.06$ & $<0.01^{*}$ \\
Re-exploration for bleeding & $2(3.07)$ & $4(5.3)$ & 0.008 \\
Postoperative Hb & $10.25 \pm 1.15$ & $12.79 \pm 1.59$ & $<0.05^{*}$ \\
Chest tube indwelling time (days) & $4.3 \pm 6.2$ & $6.7 \pm 9.2$ & $0.05^{\star}$ \\
Discharge after operation (days) & $7.2 \pm 5.4$ & $9.3 \pm 8.2$ & 0.03 \\
Postoperative morbidity & & & \\
Persistent air leakage & $8(12.3)$ & $8(10.6)$ & $>0.99$ \\
BPF/Empyema & $1(1.5)$ & $2(2.6)$ & 0.99 \\
Acute renal failure & 0.0 & 0.0 & 0.26 \\
VTE & $1(1.5)$ & $2(2.6)$ & $2(2.6)$ \\
PE & $1(1.5)$ & $1(1.3)$ & 0.26 \\
Hospital mortality & $1(1.5)$ & 0.89 \\
\hline
\end{tabular}

BPF, bronchopleural fistula; BT, blood product transfusion; $\mathrm{Hb}$, hemoglobin; PE, pulmonary embolism; TXA, tranexamic acid; VTE, venous thromboembolism. statistically significant at $P$ value $\leq 0.05$. Data were expressed as mean \pm SD or frequency as percentage.

between both groups. The incidence of persistent air leak was higher in group I (TXA group), but without any significant difference.

The overall incidence of re-exploration for bleeding and VTE/PE was higher in-group II but did not reach statistical significance. However, the amount of blood loss during the first postoperative day, days of chest tube indwelling, days of postoperative hospital stay, and postoperative empyema and/or bronchopleural fistula had significant higher incidence in group II than group I (Table 3).

\section{Discussion}

In our study, we revealed that TXA administration at a high dose at the end of surgery $(50 \mathrm{mg} / \mathrm{kg}$ as a bolus, could be repeated in half dose if needed) minimizes the total perioperative blood loss significantly in comparison with blood transfusion patients. Our results demonstrated that the amount of blood loss during the first postoperative day is significantly reduced in group I (TXA) compared with patients in group II (BT). Our returns of decreased blood loss are constant with several reports [13-15], which concluded that TXA decreased the total amount of blood loss perioperatively in adult patients who underwent elective posterior thoracic/lumbar spinal fusion surgery and in pediatric patients going through scoliosis surgery. In spite of that, the postoperative hemoglobin level was more elevated in the blood transfusion group than the tranexamic group. This could be explained by the compensatory and corrective effect of blood transfusion on the postoperative hemoglobin level. Our results demonstrate that the total incidence of VTE/PE in our cohort is $4.28 \%$, which is lower than previous studies results $[1,2]$. However, the incidence of
VTE/PE for the intergroup comparison was actually the lowest in group I (TXA). This could be explained by the scientific fact that TXA is a derivative of the amino acid lysine that reverses the hurdle of lysinebinding sites on the plasminogen particles, leading to failure of binding of plasminogen to the lysine residue on fibrin and consequently suppression of fibrinolysis [16]. This funnel of fibrinolysis results in lowering in D-dimer levels but has no effect on other coagulation parameters (e.g. platelet count, PPT, and PT). Therefore, TXA diminishes the rate at which hemostatic fibrin is liquefied, allowing for steadiness of the fibrin clot and decreased blood loss. A randomized controlled trial compared TXA with placebo in patients having coronary artery bypass grafting surgery and revealed a significant decrease in the need for reoperation owing to massive hemorrhage besides the need for any BT [17]. These results support the findings of previous metaanalysis that showed TXA decreased the pooled risk ratio or the need of blood transfusion in patients undergoing cardiac surgery compared with the controlled group [18]. The results presented in this study reveal a pattern of increased chest tube indwelling time and discharge after operation in days, which were significantly prolonged in group II (BT group), with $P$ values of 0.05 and 0.02 , respectively. We infer that prolonged chest tube insertion and prolonged hospital stay in BT group (group II) because of sepsis were associated with blood transfusion. This is concordant with previous studies [19], in which blood transfusions has been associated with increased incidence of postoperative pneumonia and sepsis with worse postoperative outcomes in various cardiothoracic surgeries. Furthermore, there were no significant differences in the occurrence of bronchopleural fistula and empyema among the groups, but the incidence was higher in group II. This finding is 
concordant with that of Thomas et al. [20] who concluded that blood transfusion increased the incidence of infectious complications by three-folds; it was also recognized as the best indicator of such complication at multivariate analysis. Lung resection surgeries all are considered demanding procedures that mostly are associated with variable degrees of blood loss owing to the accidental intraoperative venous or arterial mediastinal hemorrhage or oozing-type bleeding related to dense adhesions between lung and chest wall. Indeed, despite the beneficial effect of blood transfusion especially on hemoglobin level, concerns have been raised regarding risk of blood transfusion adverse outcomes.

\section{Financial support and sponsorship}

Nil.

\section{Conflicts of interest}

There are no conflicts of interest.

\section{References}

1 Burns PJ, Wilson RG, Cunningham C. Venous thromboembolism prophylaxis used by consultant general surgeons in Scotland. $J R$ Coll Surg Edinb 2001; 46:329-333.

2 Kakkar AK, Davidson BL, Haas SK. The investigator against thromboembolism (INATE) core group. compliance with recommended prophylaxis for venous thromboembolism: improving the use and rate of uptake of clinical practice guidelines. J Thrombo Haemost 2004; 2:221-227.

3 Nikolaou K, Thieme S, Sommer W, Johnson T, Reiser MF. Diagnosis pulmonary embolism: new computed tomography applications. $J$ Thorac Imaging 2010; 25:151-160.

4 Wang NS. Anatomy and physiology of the pleural space. Clin Chest Med 1985; 6:3-16.

5 Khalil PN, Ismail M, Kalmar P, von Knobelsdorff G, Marx G. Activation of fibrinolysis in the pericardial cavity after cardiopulmonary bypass. Thrombo Haemost 2004; 92:568-574.

6 Bedirhan MA, Turna A, Yagan N, Tasci O. Aprotinin reduces postoperative bleeding and the need for blood products in thoracic surgery: results of a randomized double-blind study. Eur J Cardiothorac Surg 2001; 20:1122-1127.
7 Sirbu H, Busch T, Aleksic I, Lotfi S, Ruschewski W, Dalichau H. Chest reexploration for complications after lung surgery. Thorac Cardiovasc Surg 1999; 47:73-76.

8 Casati V, Guzzon D, Oppizzi M, Cossolini M, Torri G, Calori G, et al. Hemostatic effects of aprotinin, tranexamic acid and amincaproic acid in primary cardiac surgery. Ann Thorac Surg 1999; 68 2252-2257.

9 Casati V, Sandrelli L, Speziali G, Calori G, Grasso MA, Spagnolo S. Hemostatic effects of tranexamic acid in elective thoracic aortic surgery: a prospective, randomized, double-blind, placebo-controlled study. $J$ Thorac Cardiovasc Surg 2002; 123:1084-1091.

10 Shaw RE, Johnson CK, Ferrari G, Brizzio ME, Sayles K, Rioux N, et al. Blood transfusion in cardiac surgery does increase the risk of 5-year mortality: results from contemporary series of 1714 propensity-matched patients. Transfusion 2014; 54:1106-13.

11 Lan N, Stocchi L, Li Y, Shen B. Peri-operative blood transfusion is associated with post-operative infectious complications in patients with Crohn's disease. Gastroenterol Rep 2018; 6:114-121.

12 Mosaad A, Abd-elfatah M, Abd-elazeem E, Arab A. A comparative study between prophylactic high dose of tranexamic acid and low dose tranexamic acid in reducing peri-operative blood loss in spine surgery. $J$ Clin Anesth Pain Med 2017; 1:1-4.

13 Wong J, El Beheiry H, Rampersaud YR, Lewis S, Ahn H, De Silva Y, et al. Tranexamic acid reduces perioperative blood loss in adults patients having spinal fusion surgery. Anesth Analg 2008; 107:1479-1486.

14 Verma K, Kohan E, Ames CP, Cruz DL, Deviren V, Berven S, et al. A comparison of two different dosing protocol for tranexamic acid in posterio spinal fusion for spinal deformity. Int J Spine Surg 2015; 19:65.

15 Sethna NF, Zurakowski D, Brustowicz RM, Bacsik J, Sullivan LJ, Shapiro F. Tranexamic acid reduces intraoperative blood loss in pediatric patients undergoing Scoliosis Surgery. Anesthesiology 2005; 102:727-732.

16 Yagi M, Hasegawa A, Nagoshi N, lizuka S, Kaneko S, Fukuda K, et al Does the intraoperative tranexamic acid decrease operative blood loss during posterior spinal fusion for treatment of adolescent idiopathic scoliosis? Spine 2012; 37:1336-1342.

17 Myles PS, Smith JA, Forbes A, Silbert B, Jayarajah M, Painter T, et al. Tranexamic acid in patients undergoing coronary artery surgery. $N$ Engl $J$ Med 2017; 376:136-48.

18 Ker K, Edwards P, Perel P, Shakur H, Roberts I. Effect of tranexamic acid on surgical bleeding: systematic review and cumulative meta-analysis. $B$ Med J 2012; 344:e3054.

19 Vamvakas EC, Carven JH. RBC transfusion and postoperative length of stay in the hospital or the intensive care unit among patients undergoing coronary artery bypass surgery: the effects of confounding factors. Transfusion 2000; 40:832-839.

20 Thomas P, Michelet P, Barlesi F, Thirion X, Doddoli C Giudicelli $R$, et al. Impact of blood transfusions on outcome after pneumonectomy for thoracic malignancies. Eur Respir J 2007; 29:565-570. 